\title{
Evidence for orbital motion of material close to the central black hole of Mrk 766
}

\author{
T. J. Turner ${ }^{1,2}$, L. Miller ${ }^{3}$, I. M. George ${ }^{1,2}$, and J. N. Reeves ${ }^{2,4}$ \\ 1 Dept. of Physics, University of Maryland Baltimore County, 1000 Hilltop Circle, Baltimore, MD 21250, USA \\ e-mail: turner@milkyway.gsfc.nasa.gov \\ 2 Code 662, Exploration of the Universe Division, NASA/GSFC, Greenbelt, MD 20771, USA \\ 3 Dept. of Physics, University of Oxford, Denys Wilkinson Building, Keble Road, Oxford OX1 3RH, UK \\ 4 Dept. of Physics and Astronomy, Johns Hopkins University, 3400 N Charles Street, Baltimore, MD 21218, USA
}

Received 10 March 2005 / Accepted 24 June 2005

\section{ABSTRACT}

Time-resolved X-ray spectroscopy has been obtained for the narrow line Seyfert galaxy Mrk 766 from XMM-Newton observations. We present analysis in the energy-time plane of EPIC pn data in the $4-8 \mathrm{keV}$ band with energy resolution $R \simeq 50$. A component of Fe K $\alpha$ emission detected in the maps shows a variation of photon energy with time that appears both to be statistically significant and to be consistent with sinusoidal variation. We investigate the interpretation that there exists a component of line emission from matter in a Keplerian orbit around a supermassive black hole. The orbit has a period $\sim 165 \mathrm{ks}$ and a line-of-sight velocity $\sim 13500 \mathrm{~km} \mathrm{~s}^{-1}$. This yields a lower limit for the central mass of $M>4.9 \times 10^{5} M_{\odot}$ within a radius of $3.6 \times 10^{13} \mathrm{~cm}(2.4 \mathrm{AU})$. The orbit parameters are consistent with higher black hole masses, but the lack of any substantial gravitational redshift of the orbit implies an upper limit to the black hole mass of $4.5 \times 10^{7} M_{\odot}$.

Key words. galaxies: active - X-rays: individual: Mrk 766 - accretion, accretion disks

\section{Introduction}

AGN are thought to be powered by accretion of material onto a supermassive black hole. The accreted gas has angular momentum, and so forms a flattened, rotating "accretion disk" as it flows toward the central black hole. Gravitational potential energy lost as the material approaches the black hole is converted into kinetic energy and radiation, with the innermost disk producing frequencies up to the ultraviolet (e.g. Haardt \& Maraschi 1993). The observed X-ray radiation is most likely produced by inverse Compton scattering of these "seed" photons by relativistic electrons close to the black hole, possibly in a corona sandwiching the disk (Haardt \& Maraschi 1993). While some of the X-ray radiation is observed directly, a significant fraction shines back onto the disk surface and produces a so-called "reflection spectrum", whose main observable feature below $10 \mathrm{keV}$ is $\mathrm{Fe} \mathrm{K} \alpha$ emission (e.g. George \& Fabian 1991).

Intrinsically narrow emission lines from an accretion disk would be affected by a combination of relativistic Doppler and gravitational redshift effects. The strong $\mathrm{Fe} \mathrm{K} \alpha$ line is emitted via fluorescence or recombination processes between 6.4-7 keV (depending on the ionization-state of the gas) and the observation of a broad and asymmetric profile was predicted for AGN (Fabian et al. 1989) before being established in data from the ASCA satellite (Tanaka et al. 1995). ASCA observations of a sample of AGN found both broad and narrow components of the $\mathrm{Fe} \mathrm{K} \alpha$ line to be commonly observed in AGN (Nandra et al. 1997). Contributions from the inner disk have long been thought to explain the observed broad component of the line while the narrow line core has been thought to come from material much further out (Fabian et al. 2000, and references therein).

Continuum components absorbed by ionized gas can account for some of the spectral curvature observed in the Fe $\mathrm{K}$ regime. It has thus been difficult to distinguish between absorbed continua and a relativistically-broadened line as explanations of some very broad spectral signatures observed in that energy-band (e.g. Reeves et al. 2004; Turner et al. 2005). With the realization that determination of disk parameters may have been compromised by confusion with absorption, it has become vital to turn to clearer signatures of the disk. Fortunately, another diagnostic has recently been discovered. Energy-shifted narrow $\mathrm{Fe}$ emission lines were first found in overlapping Chandra and XMM-Newton spectra of NGC 3516 (Turner et al. 2002). The line energies, combined with their rapid flux variations indicated these could be the long-sought "Doppler horns" of emission lines from narrow annuli on the accretion disk, perhaps illuminated by magnetic reconnection events (e.g. Merloni \& Fabian 2001) or by photon bubble 
instabilities in the inner disk (Ballantyne et al. 2005). More examples have since been reported (e.g. Guainazzi 2003; Yaqoob et al. 2003; Turner et al. 2004; Porquet et al. 2004; Bianchi et al. 2004) and the phenomenon appears to be a general property of Seyfert galaxies. A rapid (few thousand second) energy shift detected in Fe emission from the Narrow Line Seyfert 1 galaxy (NLSy1) Mrk $766(z=0.0129$; Smith et al. 1987) was of particular interest (Turner et al. 2004), strengthening the hypothesis that these lines originate very close to the central black hole. Detailed calculations of the line profiles expected from orbiting flares on the accretion disk (Dovciak et al. 2004) are consistent with observations to date, further supporting the hotspot interpretation.

Here we present an application of a relatively new analysis technique to the XMM-Newton (hereafter XMM) data from the narrow line Seyfert 1 galaxy, Mrk 766. Previous X-ray observations of Mrk 766 have shown X-ray flux variability by factors of several on timescales down to a few thousand seconds (e.g. Pounds et al. 2003). The X-ray spectrum is complex, strong features in the soft X-ray regime have been interpreted both in the context of emission from an ionized accretion disk and absorption due to ionized gas along the line-of-sight (e.g. Sako et al. 2003, and references within). Fe $\mathrm{K} \alpha$ emission has also been detected, but the detailed properties of the line have been difficult to determine (Pounds et al. 2003). Our new analysis entails plotting and statistically evaluating the data as an intensity map in the energy-time plane. Iwasawa et al. (2004) have previously analysed energy-time maps to search for time variation in emission from NGC 3516 in the $5.7-6.5 \mathrm{keV}$ range, interpreting this as accretion disk emission. In this paper we search directly for and evaluate the statistical significance of any evidence of orbiting matter close to the central black hole in Mrk 766.

\section{Energy-time analysis}

\subsection{The calculation of energy-time fluctuation maps}

$X M M$ spectra from a 2001 observation of Mrk 766 showed line emission shifting from rest-energy $5.6 \mathrm{keV}$ to $5.75 \mathrm{keV}$ within a few hours, likely to be Fe emission from very close to the black hole (Turner et al. 2004). Analysis of the mean spectrum of this AGN has shown a broad emission component from ionized Fe (Pounds et al. 2003), with equivalent width $\sim 90 \mathrm{eV}$. The breadth of the line indicates an origin close to the central black hole, supported by Pounds et al. (2003) suggestion of rapid spectral variability in the $\mathrm{Fe} \mathrm{K}$ emission.

To test the origin of rapid energy shifts in such spectral features we have created X-ray intensity maps in the energy-time plane. We have analysed XMM observations with the EPIC pn and MOS detectors made in 2001 May and 2000 May for which data reduction is described by Turner et al. (2004).

Photons from the source cell were accumulated in pixels in the energy-time plane. The pixel distribution was smoothed by the instrumental resolution in energy, given by a Gaussian of $F W H M \sim 140 \mathrm{eV}$ (appropriate for the single plus double events with the latest calibration), and by a top-hat function of width $10 \mathrm{ks}$ in time. Each time-slice was background-corrected by subtracting a time-dependent background spectrum measured in an off-source region on the same detector chip as the source (typically $\sim 5 \%$ of the source count rate). The source continuum was modelled as an absorbed power-law, of variable amplitude and slope but time-invariant absorption column density. This continuum was subtracted, leaving positive and negative residuals that comprise noise plus any emission or absorption components on top of the continuum.

Noise on this energy-time fluctuation map was calculated by propagation of Poisson shot noise errors through the smoothing process. The noise model comprises of contributions from the variable subtracted power-law component, the subtracted background component, the uncertainty in the background component and from the mean excess line emission above the continuum. Uncertainty in the determination of the variable power-law component is also present but causes a time-dependent uncertainty in the zero-level on the map of fluctuations in the region of the line rather than a random noise component in each pixel. At an energy of $6.6 \mathrm{keV}$ the fractional uncertainty in the continuum determination is \pm 0.011 which leads to a fractional uncertainty in the zero-level of the signalto-noise maps shown below of \pm 0.13 , small compared with the observed values in the Fe line. This zero-point error can cause small apparent time-variations in flux of an emission feature but cannot cause energy shifts in detected features.

The "signal-to-noise" (S/N) map presented (Fig. 1a) is the ratio of the fluctuation amplitude to the calculated noise. Formally, the noise is not normally distributed, but as the typical number of photons contributing to the noise in each smoothed pixel is $\sim 170$, the ratio may be taken as a good indicator of the statistical significance of individual smoothed fluctuations.

The smoothed energy-time map is not Fourier bandlimited, and in order to preserve the information content of the data, the maps shown for Mrk 766 are oversampled by a factor 10 on each axis. On the maps presented in Fig. 1 there are $\sim 13$ independent time bins and 28 independent energy bins, 2370 independent bins in each map and thus we expect $\sim 8$ purely statistical positive fluctuations above the $2 \sigma$ level randomly distributed across the map, significantly fewer than the actual observed number of such fluctuations.

We note here that the relatively high oversampling of the sharp-edged top-hat time-smoothing function does result in visible noise features at finer time resolution than $10 \mathrm{ks}$ : the advantage of choosing the top-hat function however is that regions on the maps that are separated by more than $10 \mathrm{ks}$ are statistically completely independent, allowing straightforward evaluation of the significance of fluctuations that are extended in time. Features from independent time bins that form part of a long-lived coherent structure have a higher significance than in an individual bin, and we discuss such features below.

\subsection{Energy-time fluctuations in Mrk 766}

Before embarking on the statistical analysis of these data, it is instructive to make a visual inspection. Figure $1 \mathrm{~b}$ shows the results for the 2001 May EPIC pn observation of Mrk 766 

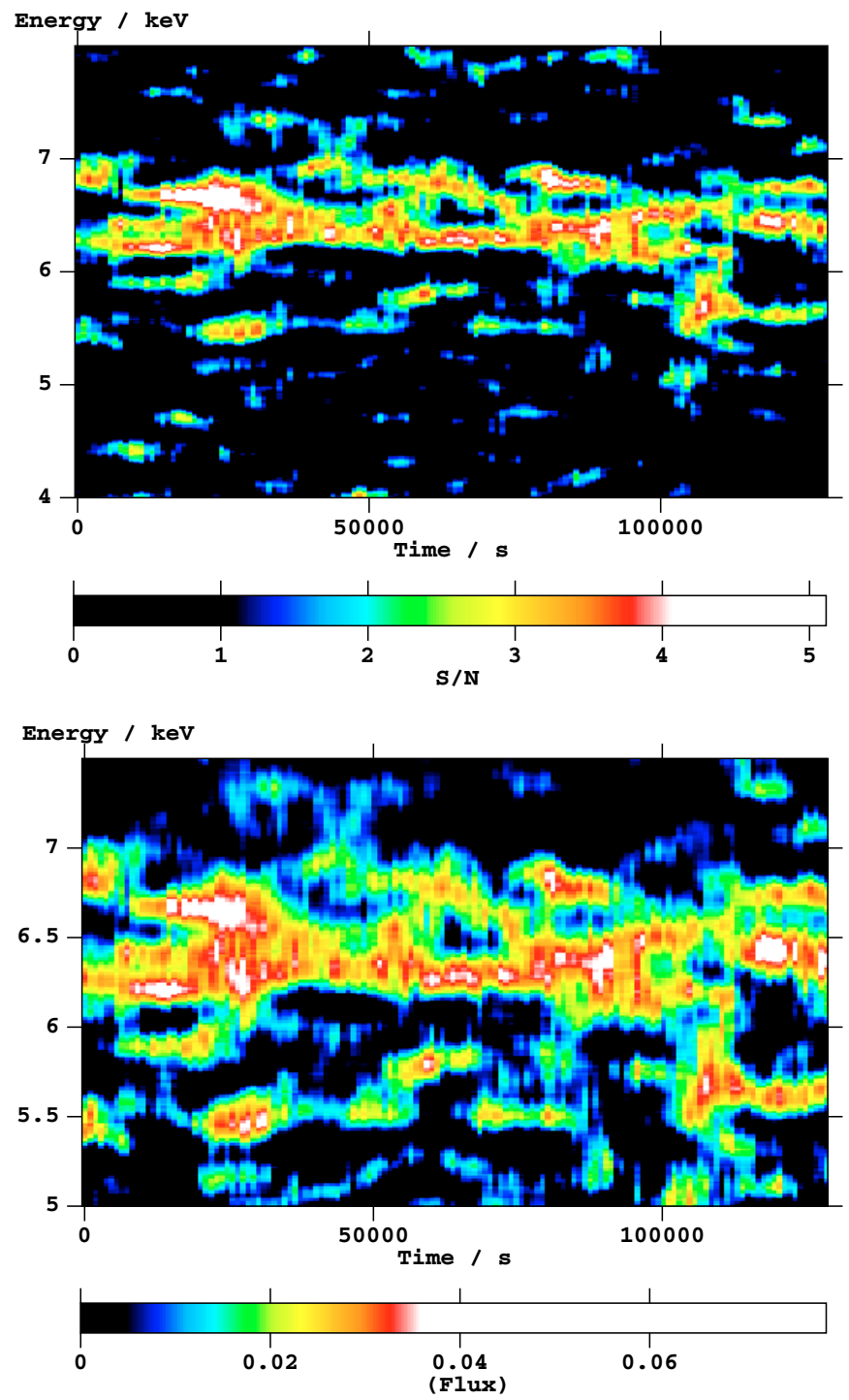

Fig. 1. a) The signal-to-noise deviations of counts in the Fe-line in the energy-time plane for Mrk 766 above the power-law continuum. The colour-scale represents excess signal in the line counts. Energy and time are oversampled by a factor 10. Data are top-hat smoothed by $10 \mathrm{ks}$ in time and Gaussian smoothed with $F W H M=0.14 \mathrm{keV}$ in energy; b) the same as a) except these are deviations in the line flux in units of count $\mathrm{s}^{-1} \mathrm{keV}^{-1}$. We note the $\mathrm{S} / \mathrm{N}$ and flux maps have different $y$-axes, as discussed in Sect. 2.2.

as a flux map, plotted as observed energy versus time. Most striking is that Mrk 766 shows emission from the K-shell of $\mathrm{Fe}$ that has the appearance of wandering in energy over a range $>1 \mathrm{keV}$. There appears to be at least one distinct coherent structure tracing out a sinusoidal trail in the energy-time domain (with mean observed energy $\sim 6.6 \mathrm{keV}$ and minimum energy occurring $\sim 60 \mathrm{ks}$ into the observation). Figure $1 \mathrm{~b}$ has a $y$-axis range that optimally displays the trail. Figure 1a shows the corresponding signal-to-noise $(\mathrm{S} / \mathrm{N})$ map with a broader range displayed on the $y$-axis to illustrate the absence of trails far away from the $\mathrm{Fe} \mathrm{K}$ regime. The highest $\mathrm{S} / \mathrm{N}$ observed is 5.2. There is no evidence for a dominant time-invariant core component of the line and no component that appears steady in energy. S/N changes are evident with time along the trails: some $\mathrm{S} / \mathrm{N}$ variations are driven by changes in the background level and/or in the flux of the underlying power-law (which varies rapidly). The most persistent feature traces a sinusoid of period $\sim 150 \mathrm{ks}$. While the assertion of a period is a bold one, especially when it is comparable to the length of the observation, in these data this is based upon coherent structures composed of many data points giving the cycle a strong definition. These maps delineate more clearly the rapid variations in flux and energy of components in the Fe K-regime reported previously by Pounds et al. (2003) and Turner et al. (2004). We also examined the corresponding $\mathrm{S} / \mathrm{N}$ map for MOS 2 (MOS 1 was in timing mode and those data were not suitable for making an energy-time map). MOS 2 is not as sensitive as the pn, with only about 30 percent of the count rate. The trails are not bright enough to be clearly detected in the MOS 2 data, but the strongest features in the pn map are confirmed by MOS 2 and therefore cannot be statistical fluctuations in the pn data. If we coadd the pn and MOS data the highest signal-to-noise ratio observed is 5.9; however, coadding these data is not our preferred method for orbit-determination owing to uncertainty in the relative energy calibration between the two instruments.

To be sure that the energy calibration is stable we examined the simultaneous variations in the peak of the instrumental $\mathrm{Au}$ edge (close to $2 \mathrm{keV}$ ) for the 2001 observation. We verified that the photon energy calibration was stable over most of the observation to $0.03 \mathrm{keV}$, with the largest variation being $0.06 \mathrm{keV}$, and that the Au-edge does not show the periodic pattern of energy shift evident in the Fe line. In any case, residual (uncalibrated) gain fluctuations could not produce the multiple-peaked structures seen in the map.

There also exists an earlier $80 \mathrm{ks}$ observation from 2000 May. That exposure begins with a pn "closed calibration", using the on-board calibration source for the first $13 \mathrm{ks}$ of the observation. Those data show the Mn-K line to be steady in peak energy. The pn instrument was then exposed to Mrk 766 for $24 \mathrm{ks}$, revealing similar characteristics to those evident in 2001.

For comparison with Mrk 766 we also show the signal-tonoise in the Fe line for NGC 5548 in the energy-time plane (Fig. 2). In contrast to Mrk 766, NGC 5548 shows no significant variations in flux or energy of the Fe line. This comparison increases our confidence that the pn instrument behaviour is well-understood and well-calibrated.

To test the interpretation of the data further we have generated a number of simulated datasets, with the same timevarying continuum and background as the real data and with a superimposed time-invariant line spectrum given by the mean line spectrum of the real data, simulated photons being generated by Poisson sampling. The simulations are conservative, in the sense that any Doppler broadening of the line is also included as a non-varying component. Visual inspection indicates that although multiple line features can be seen arising from the Poisson sampling of the broad, multiply-peaked mean line profile, long-lived trails are not visually apparent. This subjective evaluation is put onto a quantitative basis below. 


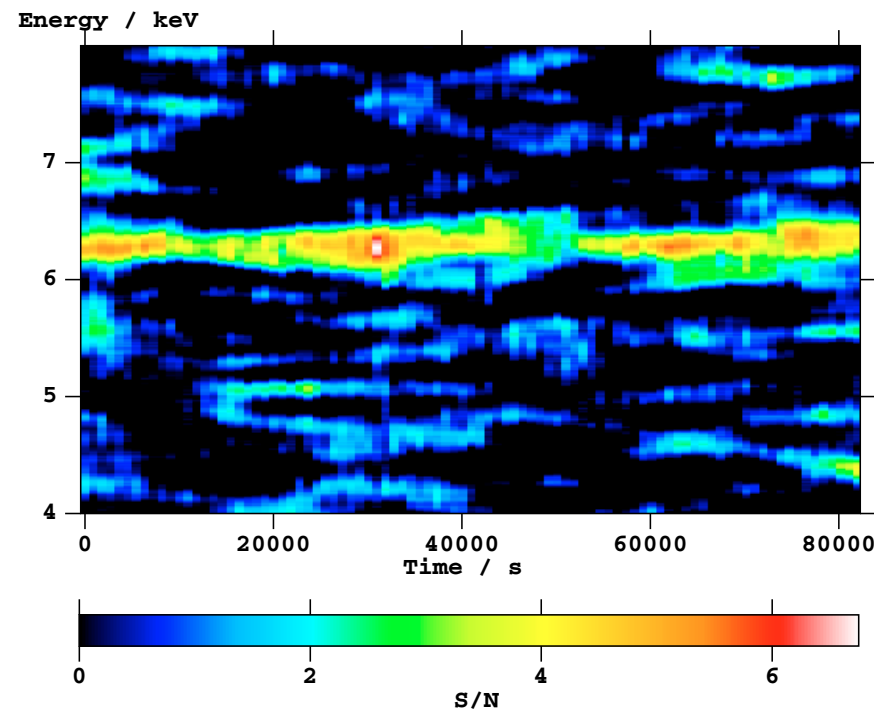

Fig. 2. Signal-to-noise in Fe line counts above the power-law continuum for NGC 5548.

\section{Statistical testing}

\subsection{Statistical methodology}

The first test one could make in attempting to look for signatures of orbiting material would be to test whether the data are consistent with the null hypothesis, that such signatures are absent. We can construct a null hypothesis by assuming that all the excess Fe emission has a time invariant spectrum, and then evaluate whether this hypothesis is acceptable by measuring the $\chi^{2}$ goodness-of-fit in independent bins in the energy-time map. The result for the Mrk 766 data is that the statistical significance (i.e. the probability of obtaining the observed result if the null hypothesis is correct) varies from $p=0.01$ to essentially unity, depending on the range of energy that is covered, the bin interval in energy, and the bin interval and placement in time. In fact, the value $p=0.01$ is only achieved for one particular placing of bins. A more typical value of $p=0.07$ is obtained if 8 equal-width time bins are used, sampling the energy range $6<E / \mathrm{keV}<7.2$ with bin width $0.14 \mathrm{keV}$. We can immediately see that there are two problems with this approach. First, if we allow the data sampling to vary until we find the most extreme value of $\chi^{2}$, then we don't know the distribution of the test statistic and hence the test is invalid. The only way around this is to choose a data sampling a priori that would be most sensitive for distinguishing between models: that sampling would depend on the velocity and period of the orbiting material, and hence already we have to introduce the concept of testing between two hypotheses ("no orbiting matter" or "orbiting matter with a particular velocity and amplitude"). Second, the $\chi^{2}$ test above is only searching for evidence of excess deviation from the model, calculated in each bin individually, above that expected from noise. There is no notion of testing for the pattern of behaviour between different bins, or for some correlation in the signal between bins. In the search for line-emitting orbiting matter, we do expect a strong correlation in the signal between adjacent time slices, with a sinusoidal variation in line energy with time. The $\chi^{2}$ test as so far discussed makes no use of such information, and as a result the probability that we would wrongly accept the null hypothesis (a "type II" error: Stuart et al. 1999) may be high (and hence the "power" of the test low).

Rather than simply testing the null hypothesis, in this paper we attempt to distinguish between two competing hypotheses: either that the $\mathrm{Fe}$ line emission is essentially time invariant, from a region that is physically distant from the central X-ray source; or that at least some of the Fe line emission arises from matter orbiting a supermassive black hole, as has been inferred from the time-averaged spectra of active galaxies as discussed in the introduction. To do this we proceed as follows.

\subsection{Searching for signatures of orbiting matter in the data}

We start by constructing models of emission that would be expected from a small line-emitting hotspot in an accretion disk, fit such a model to the time-resolved data, and hence investigate whether there is any evidence for the periodic variation of emission with time that would be expected. In reality we might expect a more complex pattern of emission: each emitting region would have a spectrum of emission rather than a single line; there could be multiple hotspots; and emitting regions could be substantially extended. In any of these cases we would expect there to be a net signal arising from the model that we have chosen to test and hence the test still has statistical power. If the emission were from a uniform disk, however, no such signature would be detectable.

In order to detect such emission, we first construct the "null hypothesis" model comprising a variable continuum and background plus a time-invariant additional component. The variable continuum is modelled as a power-law of variable amplitude and slope. In practice these quantities need to be determined from data that has been time-averaged to some extent, and here we choose a time-averaging of $5000 \mathrm{~s}$. The shorter the averaging time, the shorter the period of orbiting matter that could be searched for. But if the time-averaging were shorter than $5000 \mathrm{~s}$, the uncertainty in continuum level would become significant. This sets a limit on the range of detectable periods as described below. As described in Sect. 2.1, the continuum is assumed to be absorbed by a time-invariant cold absorber with a column density obtained from a fit to the entire dataset. The best fit absorption column density is $0.9 \times 10^{22} \mathrm{~cm}^{-2}$ and has relatively little effect at the energies of the $\mathrm{Fe} \mathrm{K} \alpha$ lines considered here. The additional time-invariant component (presumably Fe emission) is empirically determined by taking the mean of the residual spectrum after subtraction of background and absorbed power-law continuum. Hence we make no assumption about the spectrum of the time-invariant line emission: its spectrum is allowed to be determined completely by the data.

We then add a model of emission from an orbiting hotspot to the "null hypothesis" model. A component of narrow line emission whose energy varies sinusoidally with time is convolved with the instrumental $F W H M$ of $0.14 \mathrm{keV}$. The flux from 
the hotspot is modulated by the expected Doppler variation in photon arrival rate, although this is a relatively small effect at the velocities considered here. Possible distortions in the radial velocity curve arising from light-bending are neglected, which is reasonable at the radii of orbits that are detectable (as discussed below) provided any orbit is not seen very close to edge-on. The model is calculated at high time resolution and the spectrum of emission in each $5000 \mathrm{~s}$ time slice is created from that. The time-invariant Fe component is then corrected for the presence of the orbiting emission.

We may then calculate the improvement in chi-squared, $\left|\Delta \chi^{2}\right|$, that results when the hotspot is included in the model. As the null-hypothesis model assumes that all the excess Fe emission is time-invariant, this statistic tells us the improvement in the goodness-of-fit that results from including emission from orbiting material. We should note that $\left|\Delta \chi^{2}\right|$ is equal to the square of the signal to noise of the inverse-variance weighted flux in the orbit. Because of the requirement for the timeaveraged residual spectrum always to be zero, all pixels on the energy-time plane, within the energy range covered by the orbit, are affected by the addition of the model, and $\left|\Delta \chi^{2}\right|$ has to be calculated over that entire range of energy-time pixels.

The model has four free parameters: the rest-energy of the line $E$; the line-of-sight velocity amplitude $v_{\mathrm{LOS}}$; the angular frequency of the variation $\omega$; and the phase of the sinusoid $\phi$. The flux in the hotspot is assumed unknown, and in calculating $\left|\Delta \chi^{2}\right|$ for any combination of the first four parameters we adopt the flux value that maximises $\left|\Delta \chi^{2}\right|$ (for a Gaussian likelihood function this is equivalent to treating the hotspot flux as a "nuisance" parameter and marginalising over it). Only positive values of flux are allowed.

In order to evaluate the models we need to place priors on the model parameters, but the range of model parameters has to be chosen to be sufficiently broad to encompass the full range in which we would be interested. The principal Fe line emission in Mrk 766 occurs over the energy range $6.4-6.8 \mathrm{keV}$, but in general $\mathrm{K} \alpha$ emission from neutral and ionised Fe can arise over a range of energies from 6.4-6.96 keV (Verner et al. 1996). In addition, at radii close to a black hole the line energy is decreased by the gravitational redshift factor $\sqrt{1-2 r_{\mathrm{g}} / r}$, where $r_{\mathrm{g}}=G M / c^{2}$. In this paper we investigate two choices of prior on the line rest-energy. The broadest choice allows the line rest-energy in the rest-frame of Mrk 766 to vary between 6.07 and $6.96 \mathrm{keV}$, thereby covering all possible emission from radii $r>20 r_{\mathrm{g}}$ and from ionised $\mathrm{Fe}$ at smaller radii also. The second choice is the narrower range of $6.4-6.8 \mathrm{keV}$ which would be the most sensitive range for investigating the hypothesis that a significant fraction of the observed line emission comes from orbiting material.

The velocity amplitude is allowed to vary over the range $0<\beta \leq 0.15$, where $\beta \equiv v_{\mathrm{LOS}} / c$; and the phase over the range $0-2 \pi$. The necessity to time-average the data sets a limit on the maximum angular frequency that can be detected. For an energy resolution $\Delta E$, time-averaging of $\Delta t$ causes degradation of the signal when $\beta \omega \gtrsim(\Delta E / E)(\Delta t)^{-1}$, and hence we place an upper limit on $\omega$ of $2(\Delta E / E)(\beta \Delta t)^{-1}$. We also place a lower limit of $50 \mathrm{ks}$ on the period regardless of velocity. These constraints on $\omega$ and $\beta$ mean that all possible

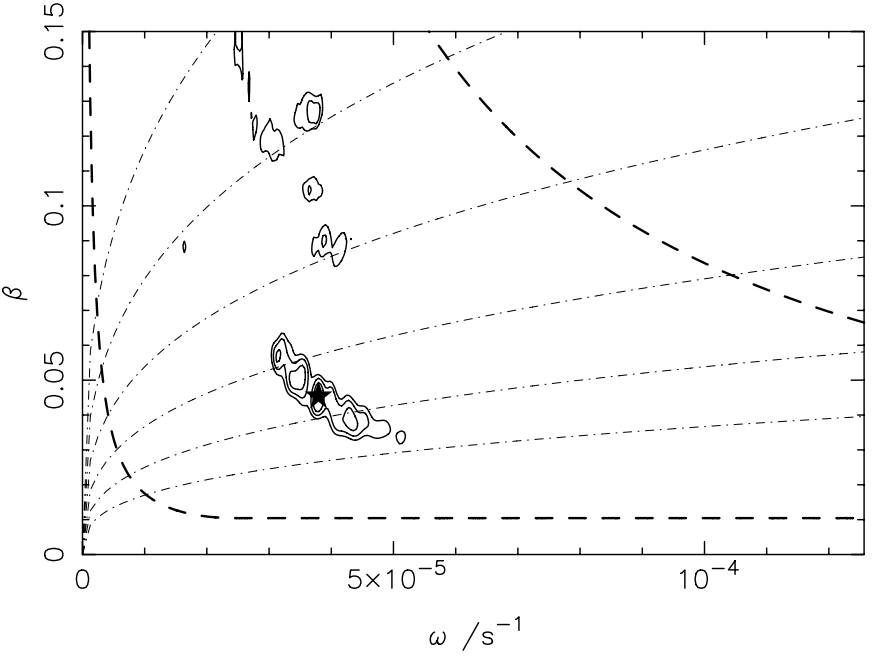

Fig. 3. Contours of $\left|\Delta \chi^{2}\right|$ in the $\omega, \beta$ plane. Contours of $\left|\Delta \chi^{2}\right|=$ $15,16,17$ are shown. The maximum value is 17.68 . The most significant orbit is indicated by a large star. The maximum values of $\omega$ and $\beta$ that have been searched place limits on the range of orbital parameters as discussed in the text. The right-side dashed line is the locus of maximum detectable $\omega$, which varies inversely with $\beta$, arising from the time-smoothing of $5000 \mathrm{~s}$. The left-side dashed line is the locus of minimum detectable energy-shift equal to the instrumental FWHM. Also shown are the expected loci of orbits of fixed inclination angle $i$ around black holes of mass covering the range $10^{5}-10^{7.5} \sin ^{-3} i M_{\odot}$ (dot-dashed lines, bottom to top respectively).

orbits that are detectable have been searched if the black hole mass is in the range $4.7 \times 10^{5}<M_{\mathrm{BH}} / M_{\odot} \sin ^{-3} i<1.2 \times 10^{7}$, where $i$ is the orbit inclination to the line-of-sight, thereby covering the possible values of black hole mass that have been suggested in the literature of $6.3 \times 10^{5} M_{\odot}$ (Botte et al. 2004), $4.3 \times 10^{6} M_{\odot}$ (Wang \& Lu 2001) and $10^{7} M_{\odot}$ (Wandel 2002), at least for high values of $\sin i$. The range of velocity amplitudes searched implies that we have searched for Keplerian orbits at $r>44 r_{\mathrm{g}} \sin ^{2} i$. The lower limit on the period implies that in the low-mass regime we have searched for Keplerian orbits at $r>137\left(M_{\mathrm{BH}} / 10^{6} M_{\odot}\right)^{-2 / 3} r_{\mathrm{g}}$. Finally, we note that there is a minimum detectable energy shift associated with an orbit, which arises when the maximum energy variation is comparable to the instrumental FWHM. Smaller energy shifts are not distinguishable from the null hypothesis of no variation with time.

These limits are displayed in Fig. 3, where the results from the fitting process are shown (adopting the broad prior on the line rest-energy). In Fig. 3, the four-dimensional parameter space is projected onto two parameters, $\omega$ and $\beta$, and contours of the maximum projected $\left|\Delta \chi^{2}\right|$ improvement are shown. The largest $\left|\Delta \chi^{2}\right|$ value is 17.68 , corresponding to a weighted signal-to-noise of 4.2 , and its location is indicated by a large star symbol on this and subsequent figures. It has values $\omega=0.38 \pm 0.02 \times 10^{-4} \mathrm{~s}^{-1}$, corresponding to a period of $165 \mathrm{ks}$, and $\beta=0.045 \pm 0.005$. The fitted orbit is shown overlaid on the pn flux map in Fig. 4. There is some degeneracy between $\omega$ and $\beta$ which arises because the orbit's period is rather longer 


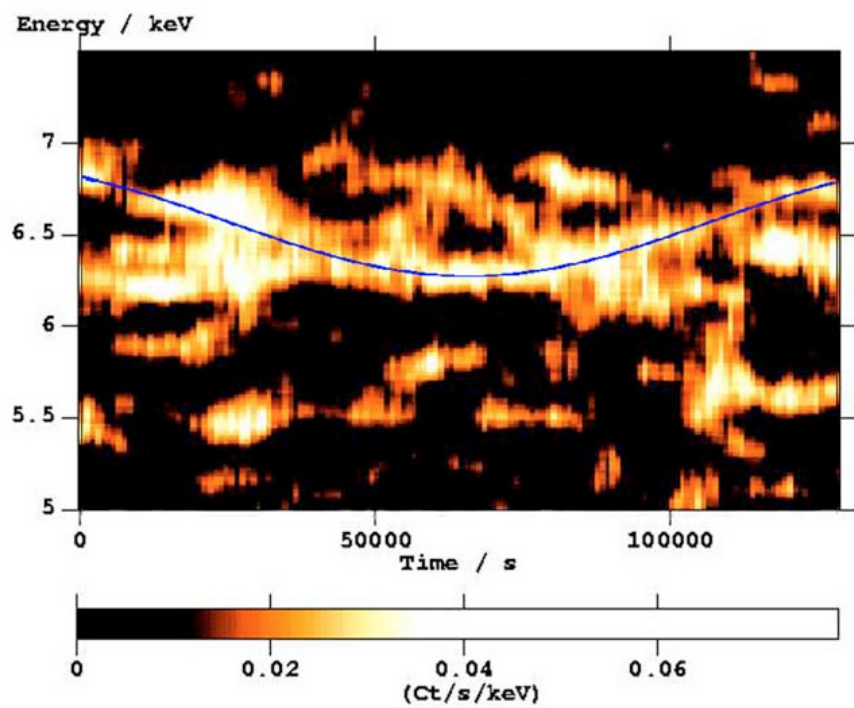

Fig. 4. The fitted orbit overlaid on the flux map from the pn data.

than the duration of the observation. Further data could help break such degeneracy.

\subsection{Simulation of the data}

The statistical distribution of $\left|\Delta \chi^{2}\right|$ is not known, however. Furthermore, the distribution varies with $\beta$, because, for a given time and energy resolution, there are many more possible ways of obtaining a positive value of $\left|\Delta \chi^{2}\right|$ at high $\beta$ than there are at low $\beta$. In order to assess the statistical significance of the candidate orbit identified above, we have created 3000 random simulations of the Mrk 766 pn observations. In creating the simulations every attempt has been made to recreate the same systematic effects present in the data. Each simulation has the same varying continuum and background as the actual data, and the same censoring of bad data. The source model consists of the "null hypothesis" model described above (absorbed time-variable power-law continuum, plus background, plus time-invariant Fe excess), and is Poisson sampled to recreate the shot noise present in the data.

\subsection{Statistical significance of candidate signatures}

These simulations are then treated as if they were real data. Each simulation is put through the same background- and continuum-subtraction and orbit-fitting procedures as the real data, and the most significant orbit that is found, as measured by the value of $\left|\Delta \chi^{2}\right|$, is recorded.

The result from this first stage of analysis is that although orbits that have high signal-to-noise (high values of $\left|\Delta \chi^{2}\right|$ ) are detected in the data, if we allow the wide range of parameters discussed above, then many of the simulations show similarly high $\left|\Delta \chi^{2}\right|$ improvements. In the case of the simulations, these do not arise from genuine orbits: they arise from the joining up of random shot noise fluctuations, and given that the shot noise in any narrow time and energy bin is high, and given that a very wide range of parameters in four-dimensional parameter space have been tested, it should not be surprising that such "random-noise orbits" are found.

However, it should nonetheless be possible to distinguish between such artefacts and a genuine orbit, because we might expect a genuine orbit to display emission continuously over a time interval comparable to the orbital period, whereas a random-noise orbit would be composed of a series of discontinuous high noise peaks that happen to lie along some path on the energy-time plane. We therefore adopt and test two measures that should be sensitive to this difference between randomnoise and genuine orbits.

The first is based on noting that, in the simulations, no actual orbits exist, and any that are found arise from linking up of random high peaks in the shot noise. So we would expect such random-noise orbits to be characterised by having a poor (high) value of $\chi_{\text {orbit }}^{2}$, where this is defined as the absolute value of $\chi^{2}$, not the improvement in $\chi^{2}$, calculated by comparing model and data along the region defined by the orbit being investigated. We expect that a genuine orbit should have a relatively low value of $\chi_{\text {orbit }}^{2}$ compared with the random-noise orbits.

The second measure is to attempt to assess the continuity of the orbit - is the orbit comprised of continuous emission, or is it comprised of a series of one or more short-lived features? Again, we expect the random-noise orbits to be discontinuous, as fluctuations between adjacent bins have no correlation. To test this, we have defined a continuity statistic, $t_{c}$, which is the maximum duration anywhere along an orbit where the flux remains at greater than half the maximum value of the orbit. In practice, the presence of noise will cause even a real orbit to appear discontinuous unless the data are smoothed, and results are presented here for smoothing by a top hat in time, with energy that follows the orbit, of three times the timeslicing employed above (i.e. a smoothing time of $15 \mathrm{ks}$ ). We could have chosen a number of subtly different ways of defining this statistic, but the choice made here was defined a priori before being tested on the data and was not modified subsequently. Alternative amounts of time smoothing were tested in order to evaluate the sensitivity of the statistic to this variable, and little change was found for time smoothing in the range 10-20 ks (for shorter time-smoothing the data are noisy, for longer smoothing timescales data and simulations appear smooth).

We now compare results from the simulations with those obtained from the data. We select from each simulation the orbit with the highest value of $\left|\Delta \chi^{2}\right|$ and measure the other two quantities, $\chi_{\text {orbit }}^{2}$ and $t_{\mathrm{c}}$. In evaluating the significance of these statistics we might be concerned that they could be correlated, so we first investigate the distribution of $\chi_{\text {orbit }}^{2}$ jointly with $\left|\Delta \chi^{2}\right|$, as shown in Fig. 5. For clarity only 1000 simulation points are shown. The candidate orbit from the real data has $\chi_{\text {orbit }}^{2}=12.36$ and $\left|\Delta \chi^{2}\right|=17.68$ : these values are also shown as a large star. In fact, there is little correlation between $\chi_{\text {orbit }}^{2}$ and $\left|\Delta \chi^{2}\right|$, but even so we measure directly the joint probability from these two statistics by constructing the two-dimensional cumulative probability distribution and measuring the fraction of points from the simulations that lie within contours of equal cumulative probability. Contours that include $0.9,0.97,0.99$ of the simulated data are shown in Fig. 5 (again, results are shown from 


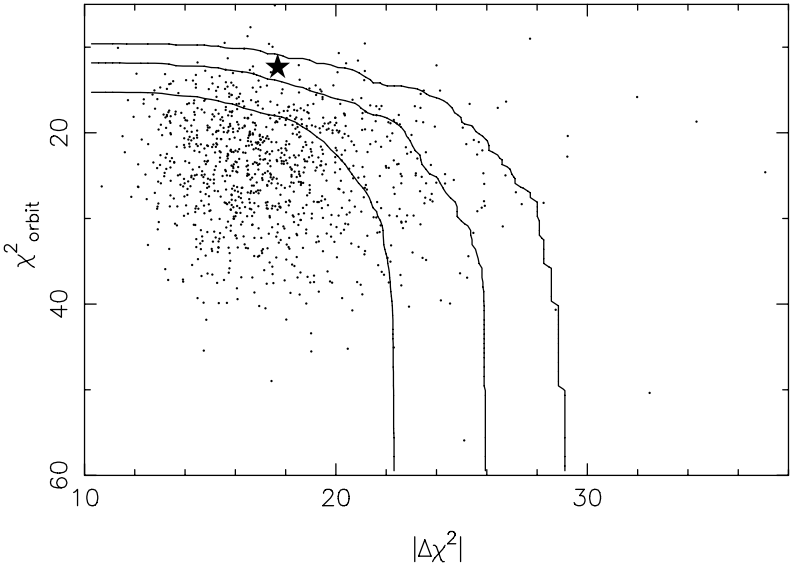

Fig. 5. Distribution of $\left|\Delta \chi^{2}\right|$ and $\chi_{\text {orbit }}^{2}$ for the orbit in each simulation with the largest value of $\left|\Delta \chi^{2}\right|$ (points), assuming the broad prior on the range of rest-energy (see text). Contours in this plane that enclose fractions $0.9,0.97,0.99$ of the points are also shown. Points deviate most from the null hypothesis towards the upper right of this diagram. Contours are derived from 3000 simulations: for clarity points are shown only for 1000 simulations. The large star denotes the values obtained for the orbit associated with the maximum in Fig. 3.

assuming the broad prior on rest-energy). The candidate orbit appears significant at a level $p=0.023$ for the $6.4-6.8 \mathrm{keV}$ range of rest-energy prior, or $p=0.036$ for the $6.07-6.96 \mathrm{keV}$ range.

We now consider the second measure, the orbit duration statistic $t_{\mathrm{c}}$. Again, we must check whether this statistic is correlated with any of the previous ones, and in Fig. 6 we show the orbit duration statistic, $t_{\mathrm{c}}$, plotted against the probability deduced from the combination of $\chi^{2}$ values on Fig. 5. There appears to be no correlation between these statistical measures, and hence we may treat the value of $t_{\mathrm{c}}$ as being an independent measure of significance. In fact, only a fraction 0.008 (narrower range of rest-energy prior) or 0.005 (broad range of rest-energy prior) of the simulations have $t_{\mathrm{c}}$ values as large as the candidate orbit (which has $t_{\mathrm{c}}$ almost equal to the maximum possible value set by the observation duration). Furthermore, only one of the random-noise orbits from the simulated data that do have long $t_{\mathrm{c}}$ values has a joint $\chi^{2}$ probability value as significant as the candidate orbit. Hence we conclude that two independent measures show that an orbit is preferred in these data compared with the null hypothesis of no time varying Fe component. The two measures yield independent significance levels of $p \simeq 0.036$ and $p \simeq 0.005$ (broad prior) or $p \simeq 0.023$ and $p \simeq 0.008$ (narrower prior) and in the remainder of this paper we shall assume that the orbit does correspond to emission from material orbiting the black hole in Mrk 766.

\section{Discussion}

\subsection{Parameters of the orbiting material}

On the assumption that the orbit found above is indeed tracing emission from material in orbit around the central black hole, we may derive some physical constraints. A lower limit

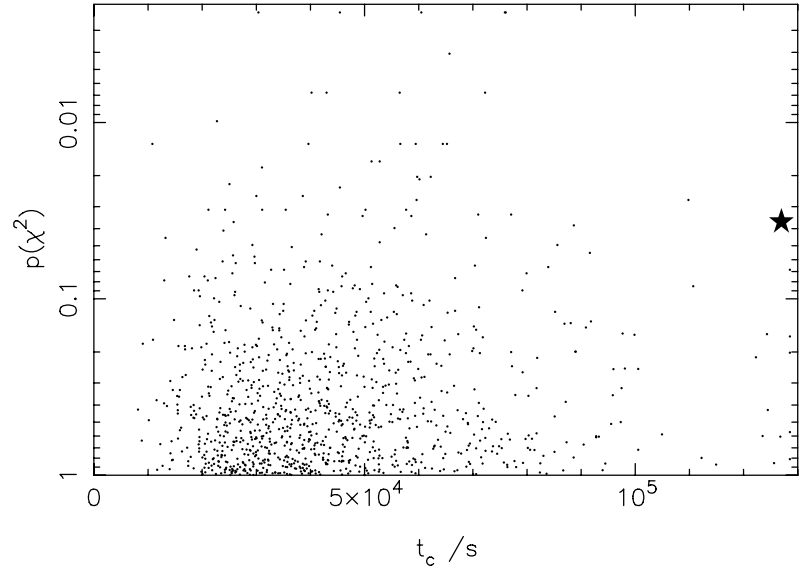

Fig. 6. Distribution of probability derived from Fig. 5 plotted against the trail duration statistic. Probability values are derived from 3000 simulations: for clarity points are shown only for 1000 simulations. The large star denotes the values obtained for the orbit associated with the maximum in Fig. 3. The broad prior on the range of rest-energy has been assumed.

to the central mass may be derived if the orbit's inclination to the line of sight is assumed to be $i=90^{\circ}$, in which case we find $M>4.9 \times 10^{5} M_{\odot}$ if we take the nominal best-fit values. At this inclination the orbital radius, assuming circular orbits, would be $3.6 \times 10^{13} \mathrm{~cm}(2.4 \mathrm{AU})$. If the central mass actually has the value $4.3 \times 10^{6} M_{\odot}$ (Wang \& Lu 2001) the orbital inclination would be $29^{\circ}$ (with an uncertainty dominated by the value of mass assumed). In this case the orbital radius would be $7.5 \times 10^{13} \mathrm{~cm}, 5 \mathrm{AU}$. Quoted in terms of the gravitational radius $r_{\mathrm{g}}$ of the black hole, the orbital radius is $r=r_{\mathrm{g}} \beta^{-2} \sin ^{2} i=494 r_{\mathrm{g}} \sin ^{2} i$, or $115 r_{\mathrm{g}}$ at $i=29^{\circ}$.

We may also place an upper limit on the black hole mass from the absence of a substantial gravitational redshift. The best-fit line rest-energy is $6.59_{-.03}^{+.08} \mathrm{keV}$ in the Earth's frame, or $6.67 \mathrm{keV}$ (coincident with the energy of the recombination line from Fe XXV), corrected to the rest-frame of Mrk 766. If we assume that the intrinsic line energy is no larger than $6.96 \mathrm{keV}$, an appropriate upper limit for $\mathrm{Fe} \mathrm{K} \alpha$ emission, this places an upper limit on the gravitational redshift. and hence a lower limit $r \gtrsim 24 r_{\mathrm{g}}$. Combined with the measured value of $\omega$ this places an upper limit on the black hole mass of $4.5 \times 10^{7} M_{\odot}$, although it is of course dependent on the correct measurement of the line rest energy. To obtain a secure determination of this quantity we need an observation that spans greater than one orbital time period in order to ensure that there is no ambiguity in the orbit fit.

\subsection{Evidence for other orbiting material}

Having established that there is evidence for some orbiting material, we may ask whether there is any evidence for any additional orbits. There are three other maxima in Fig. 3 which have $\left|\Delta \chi^{2}\right|>16$. Inspection of the corresponding orbits shows that although these are independent of the principal orbit, they are not independent of each other, and correspond to 
alternative fits to much the same fluctuations in the energy-time data. We have also measured their $\chi_{\text {orbit }}^{2}$ and $t_{\mathrm{c}}$ values. The maxima at $\beta=0.090,0.105$ have $t_{\mathrm{c}}=68435,65329 \mathrm{~s}$ with corresponding significance levels of 0.13 and 0.15 respectively, and the maximum at $\beta=0.126$ has $t_{\mathrm{c}}=30030 \mathrm{~s}$ and a significance level of 0.67 . Hence there is no evidence from this analysis that any of these possibilities correspond to genuine orbiting material. It is intriguing, however, that the $\beta=0.105$ and 0.126 fits both pass through the short-lived feature noted by Turner et al. (2004) that is discussed in the next section, and we note that if there is genuine orbiting material that is highly time-variable, as might be expected in the inner regions of an accretion disk, then our statistical tests would be biased against their detection.

It is worth noting that these orbits have very similar best-fit values of $\omega$ to that of the principal orbit that is present in the data, although differing $\beta$. They could not be coplanar with the principal orbit. Whether these orbits are genuine or arise from random noise, it might be unusual to have such a coincidence in $\omega$. In fact, the distribution of random-noise orbits is not uniform over the $\omega-\beta$ plane, and the chance of having a value of $\omega$ from a random-noise orbit within a range $\pm 0.03 \times 10^{-4} \mathrm{~s}^{-1}$ of the principal orbit's value is 7 percent, unusual but not excessively so.

Finally, we have searched for evidence of coplanar orbits. Again, there is no significant evidence of multiple coplanar orbits in the data, although the broad complex pattern of emission that is seen could be composed of a number of overlapping orbits. Further data would be required to test this hypothesis.

\subsection{Emission from material close to the black hole}

There appears to be little evidence for any longer-period components in Mrk766, there may however be shorter-period events with periods and lifetimes shorter than our minimum detectable timescale. There could be a number of reasons why such inner-disk hotspot emission does not dominate. First, we would expect hotspots only to survive at most a few orbital periods owing to differential rotation, and at small radii the orbital period is very short. Second, the necessity to time-smooth the data does result in a selection effect against detection of short time period variations as mentioned above. The highionization state of the $\mathrm{Fe}$ emission also suggests that at smaller radii $\mathrm{Fe}$ atoms may be fully ionized such that no line is possible even if the disk extends further in. Finally, the existence of a dominant component of emission at certain radii may indicate the location of a structure in the disk causing enhanced reflection at that radial location.

But signatures of inner-disk emission may be present in the data nonetheless. We note in particular one event at $t \sim 110 \mathrm{ks}$ from the start of the observation with an observed energy $\sim 5.7 \mathrm{keV}$, previously identified by Turner et al. (2004), that appears to survive $<10 \mathrm{ks}$ and that appears to be rather broad in energy. This is the signature we might expect from a short-lived flare from the inner regions of the disk. Using a more traditional method of time-resolved spectroscopy Turner et al. (2004) interpreted the data as showing a feature at $5.6 \mathrm{keV}$ that moved in energy to $5.75 \mathrm{keV}$ over a timescale of hours. However, the more informative method of data analysis employed here indicates that those two lines may be separate events and not necessarily directly related. In any case, more data are required to test the prevalence of such highlyredshifted lines.

The similarity of the mean $\mathrm{Fe} \mathrm{K} \alpha$ spectral profiles in Mrk 766 and other NLSy1s (e.g. Turner et al. 2001; Comastri et al. 2001; Romano et al. 2002) suggests the particulars of the disk in Mrk 766 may be a characteristic of the NLSy1 class and one that could give us insight into these systems. For example, a high rate of magnetic reconnection events in NLSy1s might naturally explain both their rapid X-ray continuum flux variability and the nature of the Fe line emission, one might then look for a link between high accretion rate and rate of magnetic reconnections.

\section{Summary and conclusions}

Energy-time maps from XMM observations of Mrk 766 reveal periodic energy shift in a component of $\mathrm{Fe} \mathrm{K} \alpha$ emission, with period $\sim 165 \mathrm{ks}$. This can be interpreted as evidence for emission from orbiting gas within $\sim 100 r_{\mathrm{g}}$ of the central black hole. It seems likely such material would be part of the accretion disk and in the context of that model the data may be explained as a hotspot on the disk, illuminated by magnetic reconnection. We have shown that the strongest energy trail is not likely to be produced as an artefact of shot noise. If this interpretation is correct, we obtain a lower limit for the central mass $M \geq 4.9 \times 10^{5} M_{\odot}$. The lack of a large component of gravitational redshift in the orbiting emission places an upper limit of $M \lesssim 4.5 \times 10^{7} M_{\odot}$ Significant further investigation should be possible in the near future using long $X M M$ exposures and the high-resolution AstroE2 spectra of Seyfert galaxies.

Acknowledgements. T. J. Turner acknowledges support from NASA grant NNG04GD11G. The authors thank the anonymous referee for comments that led to significant improvements to the paper.

\section{References}

Ballantyne, D. R., Turner, N. J., \& Young, A. J. 2005, ApJ, 619, 1028 Bianchi, S., Matt, G., Balestra, I., Guainazzi, M., \& Perola, G. C. 2004, MNRAS, 357, 599

Botte, V., Ciroi, S., Di Mille, F., Rafanelli, P., \& Romano, A. 2004, MNRAS, 356, 789

Comastri, A., et al. 2001, A\&A, 365, 400

Dovciak, M., Bianchi, S., Guainazzi, M., Karas, V., \& Matt, G. 2004, MNRAS, 350, 745

Fabian, A. C., Rees, M. J., Stella, L., \& White, N. E. 1989, MNRAS, 238,729

Fabian, A. C., Iwasawa, K., Reynolds, C. S., \& Young, A. J. 2000, PASP, 112, 1145

George, I. M., \& Fabian, A. C. 1991, MNRAS, 249, 352

Guainazzi, M. 2003, A\&A, 401, 903

Haardt, F., \& Maraschi, L. 1993, ApJ, 413, 507

Iwasawa, K., Miniutti, G., \& Fabian, A. C. 2004, MNRAS, 355, 1073

Merloni, A., \& Fabian, A. C. 2001, MNRAS, 328, 958 
Nandra, K., George, I. M., Mushotzky, R. F., Turner, T. J., \& Yaqoob, T. 1997, ApJ, 477, 602

Porquet, D., Reeves, J. N., Uttley, P., \& Turner, T. J. 2004, A\&A, 427, 101

Pounds, K. A., Reeves, J. N., Page, K. L., Wynn, G. A., \& O’Brien, P. T. 2003, MNRAS, 342, 1147

Reeves, J. N., Nandra, K., George, I. M., et al. 2004, ApJ, 603, 648

Romano, P., Turner, T. J., Mathur, S., \& George, I. M. 2002, ApJ, 566, 187

Sako, M., Kahn, S. M., Branduardi-Raymont, G., et al. 2003, ApJ, 596, 114

Smith, B. J., Kleinmann, S. G., Huchra, J. P., \& Low, F. J. 1987, ApJ, 318,161
Stuart, A., Ord, K., \& Arnold, S. 1999, Kendall's Advanced Theory of Statistics, Vol. 2A (London: Publ. Arnold)

Tanaka, Y., Nandra, K., Fabian, A. C., et al. 1995, Nature, 375, 659

Turner, T. J., Romano, P., George, I. M., et al. 2001, ApJ, 561, 131

Turner, T. J., Mushotzky, R. F., Yaqoob, T., et al. 2002, ApJ, 574, 123

Turner, T. J., Reeves, J. N., \& Kraemer, S. B. 2004, ApJ, 603, 62

Turner, T. J., Kraemer, S. B., George, I. M., Reeves, J. N., \& Bottorff, M. C. 2005, ApJ, 618, 155

Verner, D. A., Verner, E. M., \& Ferland, G. J. 1996, ADNDT, 64, 1

Wandel, A. 2002, ApJ, 565, 762

Wang, T., \& Lu, Y. 2001, A\&A, 377, 52

Yaqoob, T., George, I. M., Kallman, T. R., et al. 2003, ApJ, 596, 85 